\title{
Clomiphene Citrate for Induction of Ovulation in Women with Unexplained Infertility
}

\author{
Elbohoty $A^{1 *}$, El-Said $N^{1}$, Abdel-Gawad $\mathbf{M}^{1}$, Nadim $\mathbf{A}^{1}$, Kotb $\mathbf{A}^{1}$ and \\ Gozaf N ${ }^{2}$ \\ ${ }^{1}$ Department of Obstetrics and Gynecology, Ain Shams University, Egypt \\ ${ }^{2}$ Department of Obstetrics and Gynecology, El Galaa Teaching Hospital, Egypt
}

\section{Research Article}

Volume 2 Special Issue 1

Received Date: December 30, 2016

Published Date: January 09, 2017

*Corresponding author: Ahmed Elbohoty, Assistant Professor of Obstetrics and Gynecology, Faculty of Medicine, Ain Shams University, Egypt, Email: elbohoty79@gmail.com

\section{Abstract}

Objective: The aim of the current study was to compare the efficacy of CC to expectant management in women with unexplained infertility over three successive cycles.

Methods: The current randomized controlled trial was conducted at Ain Shams University Maternity Hospital. The study included women presenting with unexplained infertility for at least 12 months of unprotected regular marital life. Eligible recruited women were randomized into one of two groups: group I, including women who received Clomiphene citrate at a single daily dose of $100 \mathrm{mg}$ for 5 days; and group II, including women who were expectantly followed up without induction of ovulation. The primary outcome was the clinical pregnancy rate.

Results: A total of 113 women were enrolled in the current trial. The mean age of included women was $25.3 \pm 3.1$ years (range: 20 - 33 years). The clinical pregnancy rate was slightly, but significantly, higher in women of the CC group when compared to the control group; both per case [7/57 (12.3\% vs. 4/56 (7.1\%), respectively, p=0.357; RR $1.72,95 \%$ CI $(0.53$ to 5.55$)$; number needed to treat $(\mathrm{NNT})=19]$ and per cycle $[7 / 163(4.3 \%)$ vs. $4 / 160(2.5 \%)$, respectively, $\mathrm{p}=0.374 ; \mathrm{RR}$ $1.72,95 \%$ CI (0.51 to 5.75$)$; NNT $=56]$.

Conclusion: CC seems to be not effective in improving clinical pregnancy outcome when compared to observation alone in women with unexplained infertility.

Keywords: Clomiphene citrate; Unexplained infertility; Clinical pregnancy rate

\section{Introduction}

In spite of the advances in the evaluation and investigation for the cause of infertility, inability to conceive remains unexplained in up to $30 \%$ of wellinvestigated infertile couples [1,2]. There is no agreement on the exact definition of unexplained infertility. A diagnosis of unexplained infertility is one of exclusion. 
There is no consensus on which factors that should be excluded in order to assign the infertility as unexplained [3]. Most of clinicians agree that the basic infertility work up that should be evaluated should include semen analysis (according to the WHO criteria [4]), assessment of ovulation (using midluteal assessment of serum progesterone), and evaluation of tubal patency and uterine cavity (using the standard hysterosalpingogram or laparoscopy/hysteroscopy) [2,3,5-8]. Some clinicians add the diagnostic laparoscopy and the post coital test to that 'basic' infertility workup. There is no consensus about that, however [5-7]. In addition to this diagnostic dilemma, there are much more controversial therapeutic strategies. The reasons underlying this controversy include a lack of consistency in the literature in defining unexplained infertility, lack of a specific pathophysiological rationale that allows for a certain intervention that corrects a biological defect, the relative high probability of spontaneous conception in such couples with observation alone (which reduces the confidence of effectiveness of any intervention), and lastly the paucity of prospective randomized controlled trials that evaluate the pregnancy rates in treated and untreated couples [9].

Over the past decades, there has been a remarkable increase in the use of three main strategies: the first is superovulation (in order to develop several dominant follicles that, at least theoretically, increases the chance of fertilization and pregnancy); the second is intrauterine insemination (IUI) (which should overcome cervical factors and subtle male factors); and the third is the in vitro fertilization (IVF) as a final therapeutic modality [10]. The first of the abovementioned strategies, namely superovulation is even controversial. There is no agreement whether to induce ovulation using oral agents (clomiphene citrate [CC] or aromatase inhibitors) or gonadotropins. There have been numerous published studies on the efficacy of ovulation induction agents for women with unexplained infertility. A quite recent systematic review and meta-analysis was even published in 2010, to assess the efficacy of CC in women with unexplained infertility. Although the authors concluded that there is no evidence that CC improves pregnancy outcome in such group of women. There was a marked heterogeneity between studies that ranged between 34\% and 58\% [11]. The aim of the current study was to compare the efficacy of CC to expectant management in women with unexplained infertility over three successive cycles.

\section{Methods}

The current randomized controlled trial was conducted at Ain Shams University Maternity Hospital during the period between September 2011 and August 2012. The study protocol was in agreement to the Declaration of Helsinki for Ethical Medical Research [last updated in Korea, 2008] and was approved by the Ethical Committee of Obstetrics and Gynecology Department, Ain Shams University. All recruited women signed informed written consent after thorough explanation of the purpose and procedure of the study. All approached women had to withdraw from the study or decline without being adversely impacted regarding the medical service they should receive. The study included women attending at the outpatient infertility clinic, presenting with unexplained infertility for at least 12 months of unprotected regular marital life. Unexplained infertility was defined when there were a normal husband's semen analysis, documented ovulation and normal and patent uterine cavity and tubes [9]. A normal husband's semen analysis was defined according the WHO 2010 criteria: volume $\geq 2 \mathrm{ml}$; count $\geq 15 \times 10$ [6] per ml; normal morphology < 4\%; and progressive motility $\geq 32 \%$ [4]. Ovulation was documented with a midluteal serum progesterone $\geq 3 \mathrm{ng} / \mathrm{ml}$. Uterine cavity and tubes were assessed using either hysterosalpingogram or combined hysteroscopy/laparoscopy with chromopertubation. Women > 35 years old; those who previously received CC for more than 6 months; those who reported serious CCrelated side effects e.g. blurring of vision were not included in the study.

\section{Randomization, Allocation and Intervention}

Eligible recruited women were randomized into one of two groups: group I, including women who received CC [Clomid®, Aventis, Egypt] at a single daily dose of $100 \mathrm{mg}$ PO, starting from cycle day 2 to cycle day 6; and group II, including women who were expectantly followed up without induction of ovulation. Randomization was performed using a Computer-generated randomization system. The allocated groups were concealed in seriallynumbered sealed opaque envelops that was only opened just after recruitment. Women in both groups received the allocated treatment 30 minutes before the procedure, and were instructed not to take any form of analgesia.

Trans vaginal ultrasound scan, for measuring the endometrial thickness and the no. and average dimension of the follicle(s), was to be started on cycle day 9, and repeated every 48 hours, till reaching the size of a mature dominant follicle ( $\geq 18 \mathrm{~mm}$ ). The diameter of the follicle was taken as the average of two perpendicular 
dimensions. When mature follicle size was reached $(\geq 18$ $\mathrm{mm}$ ), intramuscular human chorionic gonadotropin (hCG) [Choriomon®, IBSA, Switzerland] was administered at a single dose of 10,000 IU. Sexual intercourse was timed on the day of triggering ovulation, and then daily for the next 3-4 days. Transvaginal ultrasound scan was repeated 48 hours after triggering ovulation to document successful ovulation (by appearance of the characteristic corpus luteum and presence of free fluid in the pouch of Douglas). Serum pregnancy test was performed 16 days after triggering ovulation. Clinical pregnancy is confirmed by positive embryonic pulsations on transvaginal ultrasound scan, performed 2-3 weeks after a positive serum pregnancy test. Transvaginal scan was performed using the $4-7-\mathrm{MHz}$ transvaginal probe Medison $\mathrm{X} 4$ Ultrasound Set [Samsung/Medison®, Seoul, South Korea]. Women who failed to get pregnant were subjected to the same study course for further 2 successive cycles.

\section{Study Outcomes}

The primary outcome was the clinical pregnancy rate, defined as sonographic detection of a viable intrauterine gestational sac. Viability was defined as presence of detectable embryonic pulsations. Secondary outcomes included the number of mature follicles, endometrial thickness at triggering ovulation, multiple pregnancy rate, as well as the medication-related side effects.

\section{Sample Size Justification}

Sample size calculation was performed using Epi Info® version 6.0, setting the type- 1 error $(\alpha)$ at 0.05 and the power $(1-\beta)$ at $80 \%$. Data from the literature showed that the spontaneous pregnancy rate in couples with unexplained infertility was 0.23 per cycle. A Cochrane systematic review ${ }^{\mathbf{1 1}}$ have showed that CC significantly increased the pregnancy rate in couples with unexplained infertility almost 2.5 folds [OR $2.5,95 \%$ CI (1.35 to 4.62)]. Calculation according to these values produced a minimal sample size 38 cases in each group. Assuming a drop-out rate of $20 \%$, at least 47 cases were required in each group.

\section{Statistical Methods}

Statistical analysis was performed using SPSS $^{\circledR}$ for Windows version 20. Difference between independent groups was analyzed using independent student's t-test (for numeric parametric variables); Mann-Whitney's Utest (for numeric non-parametric variables); and chisquared test, risk ratio and its 95\% confidence interval (for categorical variables). Yates' continuity correction was applied to the chi-squared test whenever one or more of observed values were less than 5 . Significance level was set at 0.05 .

\section{Results}

A total of 113 women were enrolled in the current trial. Figure1 shows a diagram of the study course. The mean age of included women was $25.3 \pm 3.1$ years (range: 20 33 years). The mean body mass index (BMI) was $23.8 \pm 2.6$ $\mathrm{kg} / \mathrm{m}^{2}$ (range: $19.3-32.4 \mathrm{~kg} / \mathrm{m}^{2}$ ). The mean duration of infertility was $1.7 \pm 0.5$ years (range: $1-4$ years). There were no significant differences between women of both groups regarding these initial characteristics (Table-1).

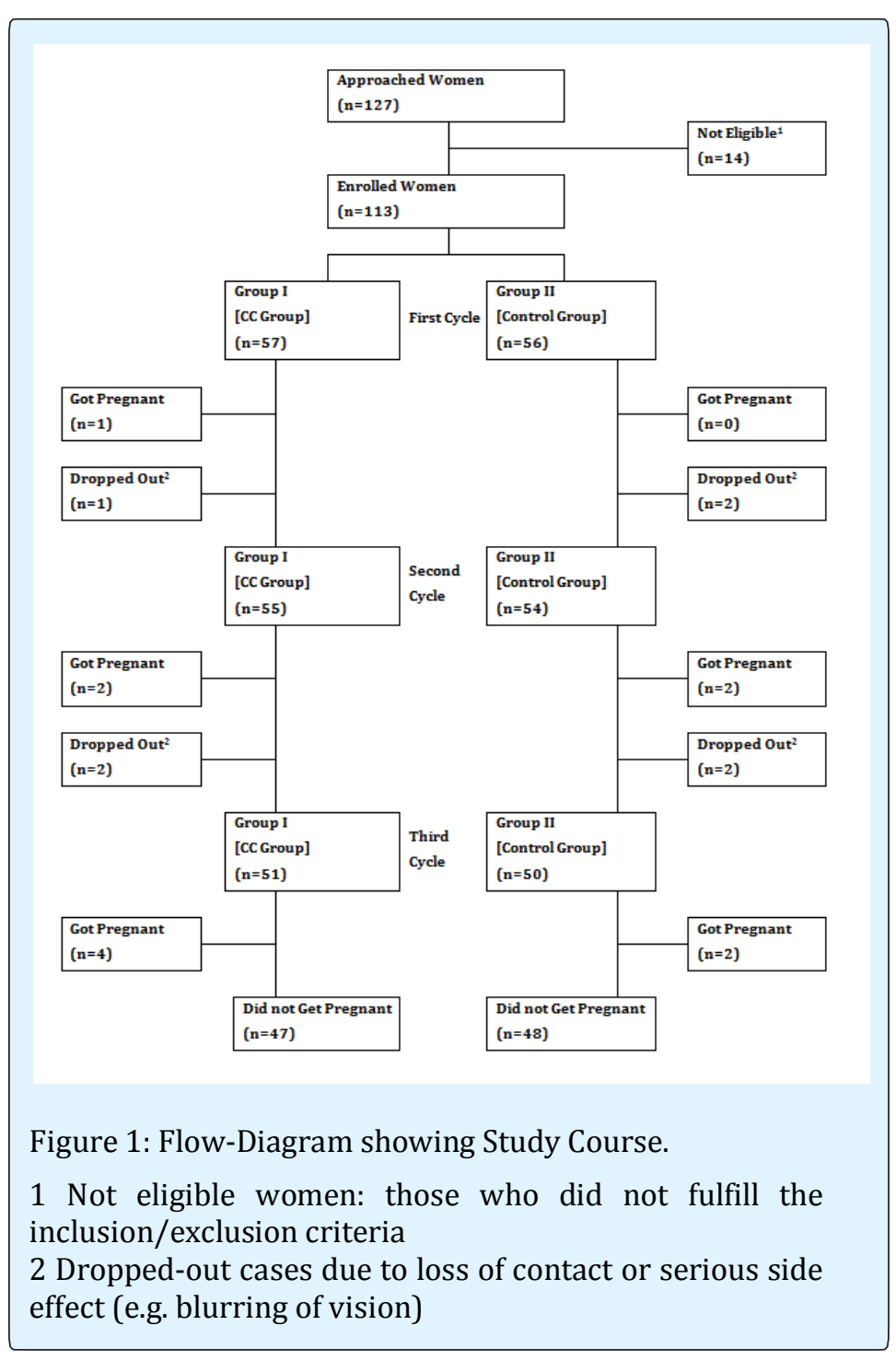




\begin{tabular}{|c|c|c|c|}
\hline & Group I & Group II & \multirow{2}{*}{ P } \\
\cline { 2 - 3 } & [CC Group] & [Control Group] & 0.74 \\
\hline Age (years) & $25.2 \pm 2.9$ & $25.4 \pm 3.1$ & 0.722 \\
\hline BMI (kg/m m $^{2}$ & $23.9 \pm 2.7$ & $23.7 \pm 2.9$ & 0.32 \\
\hline Duration of Infertility & $1.7 \pm 0.51$ & $1.8 \pm 0.49$ & \\
\hline
\end{tabular}

Table1: Initial Characteristics in Both Groups.

BMI body mass index [calculated as weight $(\mathrm{kg})$ divided by squared height $\left(\mathrm{m}^{2}\right)$ ]

Data presented as mean \pm SD

Analysis using independent student's t-test

The clinical pregnancy rate was slightly, but significantly, higher in women of the CC group when compared to the control group; both per case [7/57 (12.3\% vs. 4/56 (7.1\%), respectively, p=0.357; RR 1.72 , 95\% CI (0.53 to 5.55); number needed to treat $(\mathrm{NNT})=19]$ and per cycle $[7 / 163(4.3 \%)$ vs. $4 / 160(2.5 \%)$, respectively, $\mathrm{p}=0.374 ; \mathrm{RR} 1.72,95 \%$ CI (0.51 to 5.75); NNT = 56] (Table-2).

\begin{tabular}{|c|c|c|c|c|c|}
\hline \multirow{2}{*}{ Clinical Pregnancy Rate } & Group I & Group II & \multirow{2}{*}{ P } & RR & \multirow{2}{*}{ NNT } \\
\cline { 2 - 3 } & [CC Group] & [Control Group] & & (95\% CI) & \\
\hline \multirow{2}{*}{ Per Case } & $7 / 57(12.3 \%)$ & $4 / 56(7.1 \%)$ & 0.357 & $(0.53$ to 5.55$)$ & 19 \\
\hline \multirow{2}{*}{ Per Cycle } & $7 / 163(4.3 \%)$ & $4 / 160(2.5 \%)$ & 0.374 & $(0.51$ to 5.75$)$ & 56 \\
\hline
\end{tabular}

Table 2: Clinical Pregnancy Rate in Both Groups.

Data presented as number (percentage)

Analysis using chi-squared test

RR (95\% CI) risk ratio and its 95\% confidence interval

NNT number needed to treat

The mean endometrial thickness was higher in women of the control group when compared to women of CC group. The median no. of mature follicles was significantly higher in women of the CC group. The rates of multiple pregnancy and early miscarriage were low in included women, and were comparable in both groups (Table 3 ).
The overall rate of side effects was higher in women of CC group, but not to a significant level [ $8.8 \%$ vs. $1.8 \%$, $\mathrm{p}=0.216]$. Side effects included headache, blurring of vision, vaginal dryness and ovarian cyst formation (Table 3).

\begin{tabular}{|c|c|c|c|c|c|}
\hline & $\begin{array}{c}\text { Group I } \\
\text { [CC Group] }\end{array}$ & $\begin{array}{c}\text { Group II } \\
\text { [Control Group] }\end{array}$ & $\mathbf{P}$ & $\begin{array}{c}\text { RR } \\
(95 \% \mathrm{CI})\end{array}$ & NNH \\
\hline Endometrial Thickness (mm) & $9.4 \pm 1.3$ & $10.5 \pm 1.8$ & $<0.001^{1}$ & - & - \\
\hline No. of Mature Follicles & $2(1-2)$ & $1(1-1)$ & 0.0032 & - & - \\
\hline Multiple Pregnancy & $1(1.8 \%)$ & $0(0 \%)$ & 0.9933 & $\mathrm{NE}$ & 57 \\
\hline \multirow{2}{*}{ Early Miscarriage } & \multirow{2}{*}{$1(1.8 \%)$} & \multirow{2}{*}{$1(1.8 \%)$} & \multirow{2}{*}{0.4833} & 0.98 & \multirow[t]{2}{*}{3192} \\
\hline & & & & (0.06 to 15.33 ) & \\
\hline Overall & $5(8.8 \%)$ & $1(1.8 \%)$ & 0.2163 & 4.91 (0.59 to 40.73$)$ & 14 \\
\hline Headache & $1(1.8 \%)$ & $1(1.8 \%)$ & 0.4833 & $0.98(0.06$ to 15.33$)$ & 3192 \\
\hline Blurring of vision & $1(1.8 \%)$ & $0(0 \%)$ & 0.9933 & $\mathrm{NE}$ & 57 \\
\hline Pelvic pain & $0(0 \%)$ & $0(0 \%)$ & $\mathrm{NE}^{3}$ & $\mathrm{NE}$ & NE \\
\hline Vaginal dryness & $2(3.5 \%)$ & $0(0 \%)$ & 0.4833 & $\mathrm{NE}$ & 29 \\
\hline Cyst formation & $2(3.5 \%)$ & $0(0 \%)$ & 0.4833 & $\mathrm{NE}$ & 29 \\
\hline
\end{tabular}

Table 3: Secondary Outcomes in Both Groups. 
Data presented as mean $\pm \mathrm{SD}$; median (IQR); or number (percentage)

1 Analysis using independent student's t-test

2 Analysis using Mann-Whitney's U-test

3 Analysis using chi-squared test

RR $(95 \% \mathrm{CI})$ risk ratio and its $95 \%$ confidence interval

NNH number needed to harm

NE not estimable due to nullity in one or both groups

\section{Discussion}

Ovulatory dysfunction is one of the primary causes of reproductive failure in sub fertile couples. Women with ovulatory dysfunction do not ovulate regularly or do ovulate but fail to become pregnant. Some women ovulate regularly but fail to conceive after more than a year of unprotected intercourse and so are considered to be sub fertile. Description of the intervention Clomiphene citrate is a drug used for women with ovulatory days- function, such as luteal phase deficiency and an ovulation where there is no ovulatory cycle, or oligoovulation where there are irregular or infrequent ovulatory cycles. Clomiphene citrate usually comes as a $50 \mathrm{mg}$ tablet taken orally on the fifth through to the ninth day of the cycle. Clomiphene citrate is generally well tolerated. Side effects associated with its use include hot flashes, mood swings, headaches and visual disturbances. Many of the side effects are transitory and typically abate after the cessation of treatment. The quality and quantity of cervical mucus production in clomiphene citrate treatment cycles may sometimes be reduced but rarely to an extent that would affect the capture, transport or survival of sperm. A variety of publications have raised the question of increased ovarian cancer risks associated with Clomiphene use.

The more rigorous of these studies suggests that in women taking clomiphene for more than 12 cycles the incidence of invasive epithelial cancer increases approximately three-fold. However, it remains unclear whether this association is causal or secondary to an inherent increased risk of ovarian cancer associated with the disease processes leading to sub-fertility. How the intervention might work. The effectiveness of clomiphene citrate in the treatment of sub fertility associated with oligo ovulation has been described. It $\mathrm{h}$ as bee $\mathrm{n}$ shown to increase the number of follicles produced per cycle and, therefore, increases the potential number of eggs available for fertilisation. Clomiphene appears to act as an estrogen receptor blocker and leads to elevation of endogenous follicle stimulating hormone (FSH) production, which in turn stimulates multiple follicular development. As a result, the multiple pregnancy rate is elevated to approximately $8 \%$ to $10 \%$. Clomiphene citrate is cleared through the liver and excreted in the stools. Why it is imp ort ant to $\mathrm{d}$ o this review in oligo-ovulatory women clomiphene citrate increases the likelihood of ovulation approximately 10 fold and pregnancy approximately six-fold.

However, in the absence of a clear indication such as oligoovulation, the use of clomiphene may be considered somewhat controversial. Despite this, Clomiphene is widely prescribed in ovulatory women with unexplained infertility. Understanding the effectiveness of clomiphene in this patient group is, therefore, extremely important. The rationale for inducing ovulation in 'ovulatory' women with unexplained infertility comes from two points: first, it may overcome $\mathrm{s}$ subtle defect in ovulation not uncovered by conventional tests; and second, it may enhance the likelihood of pregnancy by increasing the number of available oocytes and thus raises the chance of successful fertilization [11]. This theoretical reasoning, however, has not met practice. Most of the published studies show no benefit from adding $\mathrm{CC}$ as a treatment for unexplained infertility; some studies show small benefit from combining it to IUI [12-17].

The current study showed a slight added benefit from prescribing CC for induction of ovulation over expectant (i.e. observation alone) management in ovulatory women with unexplained infertility; this added was not of statistically significant. From the statistical point of view, the relatively wide $95 \% \mathrm{CI}$ of the risk ratio of the clinical pregnancy rate both per case [RR 1.72, 95\% CI (0.53 to 5.55)] and per cycle [RR $1.72,95 \%$ CI ( 0.51 to 5.75 )] highlights the unpowered results regarding the clinical pregnancy rate as an outcome. Meanwhile, from the clinical point of view the NNTs for the added benefit regarding the clinical pregnancy rate of 19 (for the rate per case) and 56 (for the rate per cycle) reflects a very marginal clinical benefit. Yet, with consideration of the relative low cost of CC and the relatively low risk of serious side effects or adverse squeal (particularly the multiple pregnancy and ovarian hyper stimulation syndrome [OHSS]), this treatment may be offered to some women with unexplained infertility, but after proper counseling. A similar conclusion was made by Hughes et al. in their systematic review and meta-analysis 
conducted on 7 randomized controlled trials including 1159 women.

The authors found no significant difference in the clinical pregnancy rate between CC (with or without IUI) and placebo groups [odd ratios for CC without IUI 1.66, $95 \%$ CI ( 0.58 to 4.8 ), $\mathrm{p}=0.35$ [11]. The authors, however, emphasized that women should be counseled about the potentially almost 3 -fold increased risk of ovarian cancer with the use of $\mathrm{CC}$ for more than 12 cycles; an observation that was previously reported by Rossing [16] and Whittemore [17]. On the contrary to the results of this large systematic review, an earlier well-designed doubleblind randomized trial showed a statistically significant improvement in pregnancy rate over placebo following clomiphene citrate. This difference, however, was based on the absence of pregnancy in the placebo group of 36 patients during the four cycles of observation. In the six months that followed, seven pregnancies occurred in this group [18].

In conclusion, $\mathrm{CC}$ seems to be not effective in improving clinical pregnancy outcome when compared to observation alone in women with unexplained infertility. Nevertheless, owing to the heterogeneity in the large published systematic review and meta-analysis; and the quite conflicting results of some well-designed trials, larger trials are needed to confirm or negate this conclusion.

\section{References}

1. The Practice Committee of the American Society for Reproductive Medicine (2006) Effectiveness and treatment for unexplained infertility. Fertil Steril 86(S5): S111-S114.

2. The Practice Committee of the American Society for Reproductive Medicine (2006) Optimal evaluation of the infertile female. Fertil Steril 86(S5): S264-S267.

3. Practice Committee of the American Society for Reproductive Medicine (2008) Definitions of infertility and recurrent pregnancy loss. Fertil Steril 89(6): 1603.

4. Cooper TG, Noonan E, von Eckardstein S, Auger J, Baker HW, et al. (2010) World Health Organization reference values for human semen characteristics. Hum Reprod 16(3): 231-245.

5. Collins JA, Crosignani PG (1992) Unexplained infertility: a review of diagnosis, prognosis, treatment efficacy and management. Int J Gynaecol Obstet 39(4): 267-275.

6. Templeton AA, Penney GC (1982) The incidence, characteristics, and prognosis of patients whose infertility is unexplained. Fertil Steril 37(2): 175-182.

7. Hamada A, Esteves SC, Nizza M, Agarwal A (2012) Unexplained male infertility: diagnosis and management. Int Braz J Urol 38(5): 576-594.

8. Hatasaka H (2011) New perspectives for unexplained infertility. Clin Obstet Gynecol 54(4): 727-733.

9. Jose-Miller AB, Boyden JW, Frey KA (2007) Infertility. Am Fam Physician 75(6): 849-856.

10. Kamath MS, Bhattacharya S (2012) Demographics of infertility and management of unexplained infertility. Best Pract Res Clin Obstet Gynaecol 26(6): 729-738.

11. Hughes E, Brown J, Collins JJ, Vanderkerchove P (2010) Clomiphene citrate for unexplained subfertility in women. Cochrane Database Syst Rev 20(1): CD000057.

12. Crosignani PG, Walters DE, Soliani A (1991) The ESHRE multicentre trial on the treatment of unexplained infertility: a preliminary report. European Society of Human Reproduction and Embryology. Hum Reprod 6(7): 953-958.

13. Guzick DS, Carson SA, Coutifaris C, Overstreet JW, Factor Litvak P, et al. (1999) Efficacy of superovulation and intrauterine insemination in the treatment of infertility. National Cooperative Reproductive Medicine Network. N Engl J Med 340(3): 177-183.

14. Goverde AJ, McDonnell J, Vermeiden JP, Schats R, Rutten FF, et al. (2000) Intrauterine insemination or in-vitro fertilisation in idiopathic subfertility and male subfertility: a randomised trial and costeffectiveness analysis. Lancet 355(9197): 13-18.

15. Aboulghar MA, Mansour RT, Serour GI, Al Inany HG (2003) Diagnosis and management of unexplained infertility: an update. Arch Gynecol Obstet 267(4): 177-188.

16. Rossing MA, Weiss NS, Daling JR, Moore DE, Self SG, (1994) Ovarian tumours in a cohort of infertile women. N Engl J Med 331(12): 771-776. 
17. Whittemore AS, Harris R, Itnyre J (1992) Characteristics relating to ovarian cancer risk: Collaborative analysis of 12 U.S. case controlled studies. Am J Epidemiol 136(10): 1184-1203.

18. Glazener CM, Coulson C, Lambert PA, Watt EM, Hinton RA, et al. (1990) Clomiphene treatment for women with unexplained infertility: placebo-controlled study of hormonal responses and conception rates. Gynecol Endocrinol 4(2): 75-83. 